\title{
Evaluation of a new pan-enteric video capsule endoscopy system in patients with suspected or established inflammatory bowel disease - feasibility study
}

\section{다(1) $(9)$}

\author{
Authors \\ Rami Eliakim¹, Cristiano Spada ${ }^{2,3}$, Alon Lapidus ${ }^{4}$, Inbal Eyal ${ }^{4}$, Silvia Pecere ${ }^{5}$, Ignacio Fernández-Urién ${ }^{6}$, Adi Lahat ${ }^{1}$, \\ Guido Costamagna ${ }^{2,3}$, Avraham Schwartz ${ }^{7}$, Yulia Ron ${ }^{8}$, Henit Yanai ${ }^{8}$, Samuel Adler ${ }^{7}$
}

Institutions

1 Sheba Medical Center, Gastroenterology, Ramat Gan, Israel

2 Catholic University - Digestive Endoscopy Unit, Rome, Italy

3 Fondazione Poliambulanza Istituto Ospedaliero Digestive Endoscopy, Brescia, Italy

4 Medtronic Inc, Yoqneam, Israe

5 Policlinico Universitario Agostino Gemelli - Digestive Endoscopy Unit, Rome, Italy

6 Complejo Hospitalario de Navarra - Servicio de Digestivom Pamplona, Spain

7 Shaare Zedek Medical Center - Gastroenterology, Jerusalem, Israel

8 Tel Aviv Sourasky Medical Ceneter - Gastroenterology, Tel Aviv, Irael

submitted 5.3.2018

accepted after revision 30.5.2018

Bibliography

DOI https://doi.org/10.1055/a-0677-170 |

Endoscopy International Open 2018; 06: E1235-E1246

(c) Georg Thieme Verlag KG Stuttgart · New York

ISSN 2364-3722

Corresponding author

Rami Eliakim, Sheba Medical Center - Gastroenterology,

Tel-hashomer Ramat-Gan 5265601, Israel

Fax: +972-3-5305901

abraham.eliakim@sheba.health.gov.il

\section{ABSTRACT}

Background and study aims Inflammatory bowel disease (IBD) affects the small bowel and colon. Endoscopic evaluation of these organs is essential. The new pan-enteric Crohn's capsule (PCC) system is customized for complete coverage of IBD lesions in the entire bowel, allowing assessment and follow-up of disease severity and extent. The aim of this study was to evaluate the functionality of the PCC system in patients with suspected or established IBD.

Patients and methods This was a prospective five-center feasibility study assessing the performance of PCC. Subjects ingested PCC after patency assurance with standard bowel preparation plus boosts. The primary endpoint was successful procedure, that is, video creation and report generation in accordance with methodology. Secondary endpoints were subjective coverage of the entire bowel, duration of reading time, video quality and occurrence of adverse events.

Results Forty-one patients were included in the study with a mean age of 40.8 years $\pm 15.5,46 \%$ of whom were males. Seventy-one percent of patients had established Crohn's disease (CD) and 53\% had active disease. Cleansing was graded good/excellent in $95 \%$. All 41 videos met the primary endpoint. There was no retention, $83 \%$ reached the toilet while still recording. Thirty-one percent of patients with $C D$ had proximal disease. Bowel coverage was graded $6.7 \pm 0.6$ and $6.1 \pm 1.3(1-7$, unconfident-confident), image quality $6.1 \pm 0.8(1-7$, poor-excellent), and reading time $3.7 \pm 1.4$ ( $1-7$, very short to very long).

Conclusions The PCC system is a minimally invasive system allowing extensive evaluation of the entire bowel in patients with IBD.

\section{Introduction}

Crohn's disease (CD) and ulcerative colitis (UC) are chronic inflammatory bowel diseases affecting the whole gastrointestinal tract or the colon [1]. CD affects the small bowel (SB) and colon in about $50 \%$ of patients, SB only in $33 \%$, and colon only in $20 \%$ of patients [2-3]. Recent studies using SB capsule endoscopy have reported up to $66 \%$ proximal SB involvement in patients with established $C D$ and that it carries poor prognosis [4-7]. Moreover, similar to the large bowel, Kopylov et al have shown that up to $85 \%$ of patients with established CD in clinical remission have active endoscopic SB disease, $5 \%$ to $21 \%$ have moderate-severe disease, and only $14 \%$ are in deep remission.[8] 


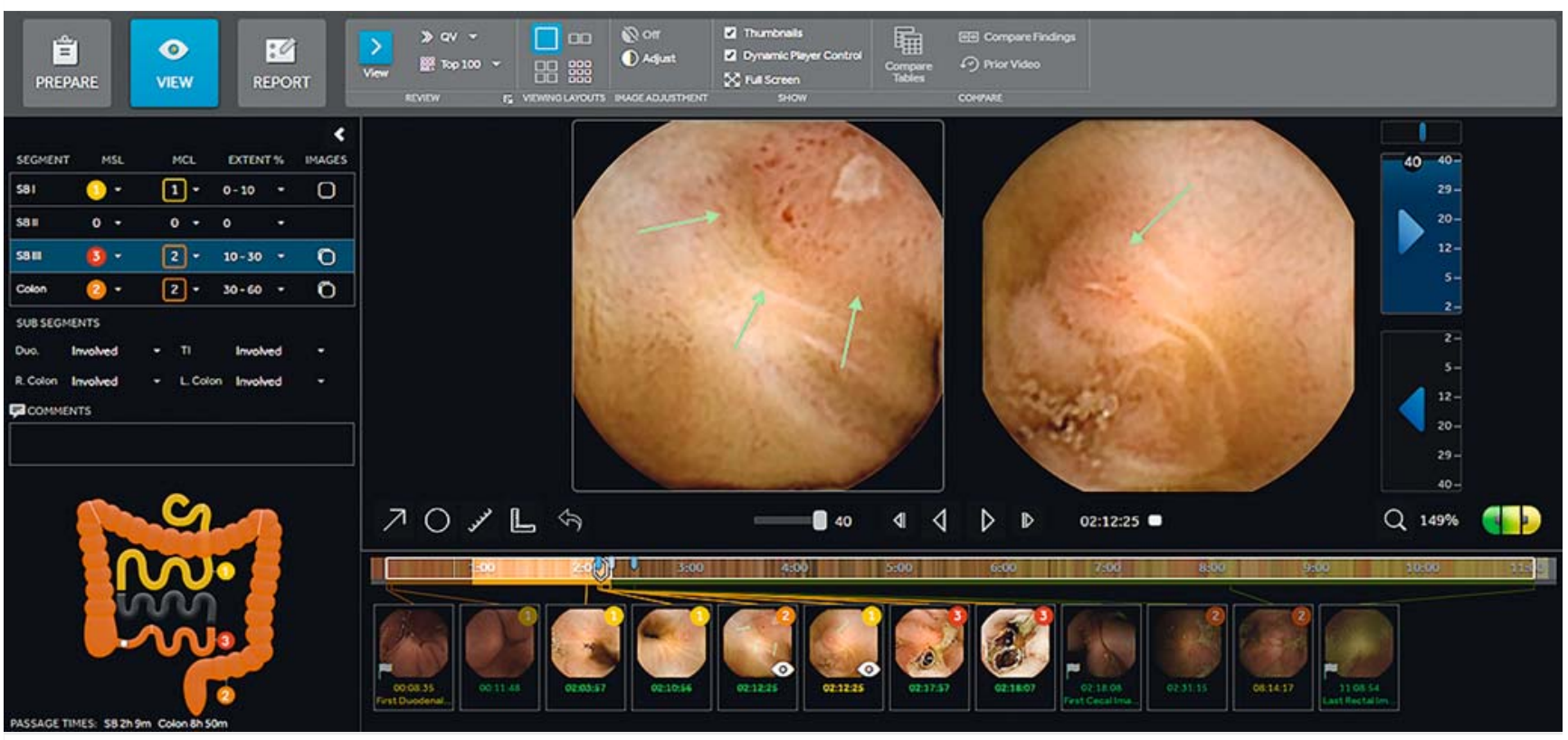

Fig. 1 PillCam Crohn's system representative image of gastrointestinal table and map.(Source: Medtronic)

In recent years, a significant effort has been made to identify patients and categorize them into low versus high risk, and accordingly treat them step up or top down, as well as to explore the concept of achieving deep remission and mucosal healing [1]. Targets have been suggested by the international organization for IBD to achieve these goals: the treat to target concept [9].

While the large bowel is easily accessible with colonoscopy, the SB is not easily accessible to optical endoscopy and is usually visualized via cross-sectional imaging or SB capsule endoscopy. Patients with $C D$ are somewhat reluctant to undertake these procedures as they undergo many colonoscopies and cross-sectional imaging over the years. Recently, a few studies have been reported which used the PillCam Colon 2 (CC2) system (Medtronic, Yoqneam, Israel), while disengaging its 2-hour sleep mode, to visualize the small and large bowel in patients with IBD [10-13]. Thus, the stage was set for a pan-enteric PillCam Crohn's Capsule (PCC) that can supply critical information on pan-enteric disease distribution and burden. This has the potential to aid in patient classification into low versus high risk, as well as to aid in patient monitoring and assess whole-gut mucosal healing/response to therapy [13-16].

Concurrently, a new PCC system has been developed and released. This includes a pan-enteric wireless capsule endoscope optimized for efficient visualization of inflammatory lesions and a novel assessment platform designed specifically to address IBD patient management and comprehensive mucosal assessment over time. This includes a new mode to describe and assess disease severity and extent ( $\bullet$ Fig. 1 ) as well as options to report on patient treatment over time ( $\triangleright$ Fig.2), review prior studies ( $\triangleright$ Fig.3) and allow clinical and endoscopic follow-up of the patient. Thus, the PCC system may be used to continuously monitor CD mucosal inflammation over time.
The primary objective of this five-center prospective feasibility study was to evaluate the functionality of this new system (capsule and software) in patients with established or suspected IBD. The secondary objectives of this study were to assess the PCC system coverage of colon and SB, overall duration of reading time, quality of images and side effects of the procedure.

\section{Patients and methods}

\section{Study design}

This was a prospective, single-arm, feasibility study conducted at five separate study sites in Europe (Spain and Italy) and Israel between July 2016 and February 2017. The study protocol was approved by the IRB/Ethics Committee of all participating clinical sites and was conducted in accordance with the Declaration of Helsinki. All subjects or parents gave written, informed consent prior to data collection and study procedures. (Clinicaltrials.gov, NCT02742714, March 2016)

Sixty-eight subjects were screened and 14 subjects were categorized as screen failure (patency failure). Fifty-four subjects were enrolled into the study, five withdrew consent, and eight had procedure-related protocol deviations. Forty-one subjects were included in the final analysis ( $\triangleright$ Fig.4). Bowel preparation was similar to that of patients undergoing colon capsule procedures, followed by PCC capsule ingestion, PCC system case review and follow-up. Patency capsule examination preceded PCC system ingestion for all established Crohn's subjects.

\section{Study participants}

All subjects that were eligible for the study were required to meet the inclusion criteria at screening which included: age 18 to 75 years and known or suspected IBD. A complete listing of inclusion/exclusion criteria ( $\triangleright$ Supplemental Table 1 ). 


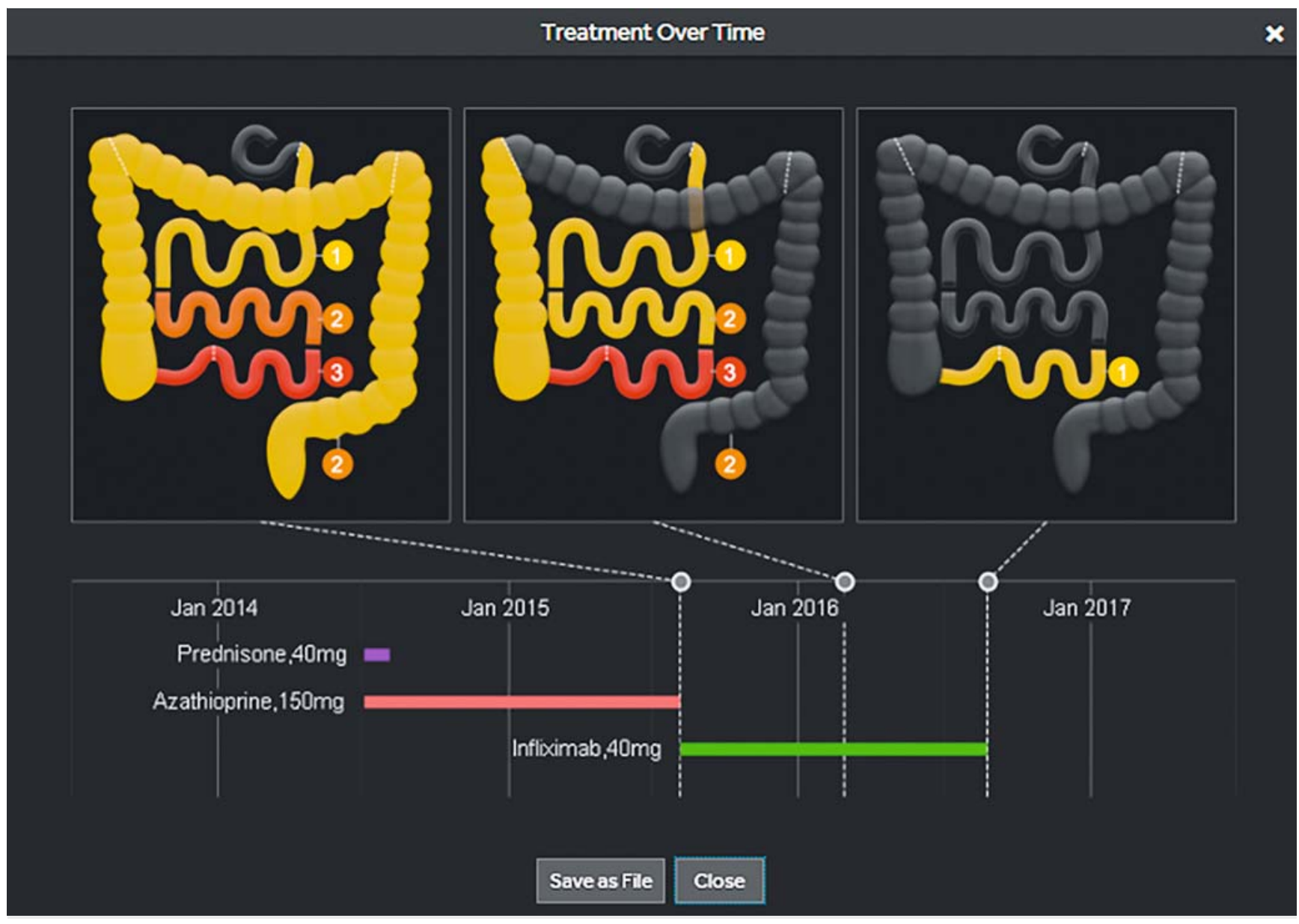

- Fig. 2 Treatment over time visualization using the PillCam Crohn's system. (Source: Medtronic)

\section{Test device}

The PillCam Crohn's system (Medtronic, Yoqneam, Israel), is composed of four main subsystems: (1) the PillCam Crohn's ingestible capsule; (2) PillCam Recorder (DR3); (3) PillCam Software (Version 9.0); and (4) Given Workstation. The PCC system is fully compliant with all safety and radio standards and regulations similar to the currently marketed PillCam endoscopy systems and received the CE mark (2016) and clearance from the US Food and Drug Administration clearance (2017).

During passage of the PCC capsule throughout the gastrointestinal tract, the DR3 receives and interprets real-time input from the capsule and provides audiovisual guidance to patients throughout the procedure. The PCC capsule itself is a twoheaded capsule with a field of view of 344 degrees, along with an adaptive frame rate technology which obtains up to 35 frames per second adapting to the speed of transit, allowing better tissue coverage and battery conservation. The capsule starts photographing once ingested, thus extensive visualization of the SB and the colonic mucosa is achieved, with increased sensitivity for visualization of inflammatory lesions and their representation in the procedure video. The PCC capsule has a minimum battery time of 10 hours, and usually works for 12 hours.
The PCC system platform and software incorporates a novel assessment methodology for inflammatory disease, specifically CD. This method results in a comprehensive, yet simple and quick, standardized qualitative assessment of the mucosal inflammatory status of the patient's pan-enteric disease. The software divides the SB portion of the procedure video into three approximately equidistant segments, in terms of tissue length and not transit time, as well as the colon. Three key assessment parameters are then assessed: disease distribution, lesion severity and linear extent.

Disease distribution refers to which of the four segments are involved in the inflammatory process. Lesion severity is assessed using two assessment parameters, per each of the segments: The Most Severe Lesion and the Most Common Lesionreferring to the severity grade of the most severe lesion and of the predominant lesions, respectively. Severity of lesions is graded based on a three-tier severity grade incorporated into the assessment. Linear extent is also assessed per segment and refers to estimation of the percentage of tissue involved linearly in the inflammatory process out of the entirety of the segment. In addition, four anatomical regions with clinical or therapeutic significance are assessed and reported on.

To reduce assessment time, video review is conducted on both of the capsule heads simultaneously, at a relatively high 


\section{(v) PRIOR STUDY: $\quad$ CROHN'S -}

FINDINGS: Patient 3 Findings

\begin{tabular}{|c|c|c|c|c|}
\hline SECNGNT & MSL & $\mathrm{MCL}$ & EXTENT\& & IMACES \\
\hline 581 & & 2 & $10-50$ & \\
\hline $58=$ & (2) & 2 & $10-30$ & \\
\hline $58=11$ & 3 & 3 & $50-60$ & \\
\hline Colan & & $\mathbf{2}$ & $0=10$ & \\
\hline
\end{tabular}

SUBSECMENTS

Duo. Notimained $\pi$ In Inolved
R.Colan Invared LCobn Notincived

\section{FREASONFORREFERRAL:}

18y.o. male with known Crohn's Disease for 2.5 years. Based on his capsule study a hair yearago, Azathioprine treatment was replaced by inficimsb szomg every s weeks. He is referred topilcam Crohn's stuoy in order to assess response to 6 months of Infiximab.

\section{FPROCEDUREINFORYATIONAND FINOINGS:}

capsute provideda milexaminstion of whole smal boweland colon with adequate deansing.

\section{SMALL BOWEL: Intiammatory imoivement of allS S8}

\section{segments:}

In S81-mild severity úcers limited to $10-50 \%$ of the segment Duodenum wes not imohed.

In S82-precominanty/mila severity úcers alongsice moderate severity ulcers, imited to $10-50 \%$ of the segment?

In 585-severe infammstion inwaving so-60s of the segment, incuding the Terminal teum.

Nostricture was detected.

colON: Mid-moderate severity ulcers imoling < $20 \%$ of colon and imited to theright colon. The rest of the colon Was nombl

Fig. 3 Feature of the PillCam Crohn's system to review prior patient studies. (Source: Medtronic) 
Screened $(N=68)$

Screened failure $(\mathrm{N}=14)$

Enrolled $(\mathrm{N}=54)$

Withdrew $(\mathrm{N}=5)$

Full analysis set $(\mathrm{N}=49)$

Procedure related protocol deviations $(\mathrm{N}=8)$

Per protocol analysis set $(\mathrm{N}=41)$

- Fig. 4 Study patient flow diagram for PillCam Crohn's capsule evaluation.

review speed while adhering to the method of "escalating severity" assessment: only spending time on categorizing and capturing thumbnails of the lesion with the highest-ranking severity encountered thus far in the segment. This method qualitatively assesses overall mucosal disease severity. This is presented in a very simple but comprehensive graphical representation of the patient's bowel, both in a colored table and a schematic gastrointestinal map ( $\triangleright$ Fig. 1 ), and is summarized in a report ( $\triangleright$ Fig. 5).

Due to standardization and simplicity of this assessment, the methodology has the capacity to aid physicians in comprehensively and continuously monitoring their patients' disease progression and dynamics, assessing responsiveness to treatment and making informed decisions at management crossroads. This review method was conducted in the trial. Each of the trial centers underwent specific training with the new system and assessment method to arrive at uniform proficiency.

Finally, because this novel PCC system divides the SB into segments according to capsule progression and not by SB transit time, we looked to see among the established CD patients whether and at what percentage there is proximal SB involvement.

\section{Study procedure}

Screening visits were completed within 30 days prior to the PCC procedure. Screening assessments included meeting inclusion/ exclusion criteria, medical history and concomitant medications, previous gastrointestinal procedures, pregnancy test (if applicable), and signing informed consent. Patients with known $\mathrm{CD}$ were required to prove patency by the patency capsule (or another approach) per the discretion of their physician. Patients were required to attend two study visits, the initial visit for screening and the PCC procedural visit, which was followed by a phone call 5 to 9 days later.

One day prior to the PCC procedure, standard bowel prep was initiated. That included a clear liquid diet and administration of a purgative sulfate-free polyethylene glycol (PEG) elec- trolyte lavage (SF-ELS) solution including either Meroken (Taro Pharmaceutical Industries Ltd, Haifa, Israel) or Isocolan (Sigmar Italia S.r.l., Almè-Bergamo, Italy) or Solución Evacuante Bohm (Laboratorios Bohm, Madrid, Spain) divided into two doses: $2 \mathrm{~L}$ on the evening before the exam and $2 \mathrm{~L}$ on the morning of the exam day as well as laxative boosts (SUPREP [Braintree Laboratories, Inc., Braintree, MA, USA]/Pico-Salax [Ferring GmbH, Kiel, Germany)]/Eziclen [Ipsen Pharma, Barcelona, Spain]/Izinova [Beaufour Ipsen Industrie, Dreux, France]) during the ingestion day. The complete bowel prep procedure is described in $\downarrow \mathbf{T a}$ ble 1.

Once bowel prep was complete, subjects ingested the PCC. One hour later, an optional dose of $10 \mathrm{mg}$ metoclopramide was taken only if the capsule remained in the stomach. Upon SB detection, subjects were then required to take an additional volume of laxative which consisted of 0.5 bottle $(88 \mathrm{~mL})$ of Suprep or 1 sachet of Pico-Salax ( $10 \mathrm{mg}$ sodium picosulfate) to maintain adequate colon cleansing and augment capsule propulsion. A second boost of laxative (as mentioned previously) was administered 3 hours after the first boost. If the capsule was not excreted after this time, subjects were asked to use a $10 \mathrm{mg}$ bisacodyl suppository or a third boost of laxative according to the investigator's decision.

Throughout the procedure, subjects were instructed to verify when the capsule was excreted and to document the time of exit. Once the procedure was complete, the raw data and video were downloaded from the DR3 to the workstation. Subjects were then followed-up 5 to 9 days later to assess their wellbeing and collect information on adverse events (AEs).

\section{Capsule endoscopy and colonoscopy video evaluation}

All subjects underwent bowel preparation, followed by PCC examination. The videos were then evaluated by a local site reader. All evaluators were familiar with PCC and passed the PCC training session conducted by the study sponsor.

\section{Outcomes measures}

The primary objective of this study was to evaluate the PCC system functionality in established and suspected IBD subjects. Secondary objectives included reader assessments of PCC coverage of the SB and colon, overall study reading time, capsule video quality, and safety of the system, all of which were conducted by answering a subjective questionnaire [17].

The primary endpoint was successful PCC procedure in terms of video creation and report generation per training instructions. Procedure success was defined as creation of both PCC video and report and the indication from the reader that the report included the information ( 3 points and up on a 5 point scale, coded as "1-No information" to "5-Full information") that he/she intended to report on. After each read, the readers were asked to complete a subjective questionnaire, which included system performance assessment, if there were any problems encountered during video review and report generation as well as if the report included all the information that was intended to report on. 


\title{
Capsule Endoscopy Report
}

\section{PillCam Crohn's}

Patient Name
ID
Birth Date
Gender
Capsu'e ID
Procedure Date

\begin{abstract}
Reason for Referral
85 y. O. femsle with Crohn's Disesse for 40 yesrs. Bssed on her lsst espsule study 6 months sgo, she received one month's tres:ment with PREONISONE $40 \mathrm{mg}$ with tspering down. In sddition. Azsthioprine trestment was replsced by Infiximsb $300 \mathrm{mg}$ every 8 weeks.

She is still symptomste. She is referred to PillCam Crohn's study in order to sssess a response to Inflocimsb trestment.
\end{abstract}

\section{Patient Data}

Small bowel passage time: $3 \mathrm{~h} 4 \mathrm{~m}$, Colon passage time: in $7 \mathrm{~m}$ Procedure Information and Findings

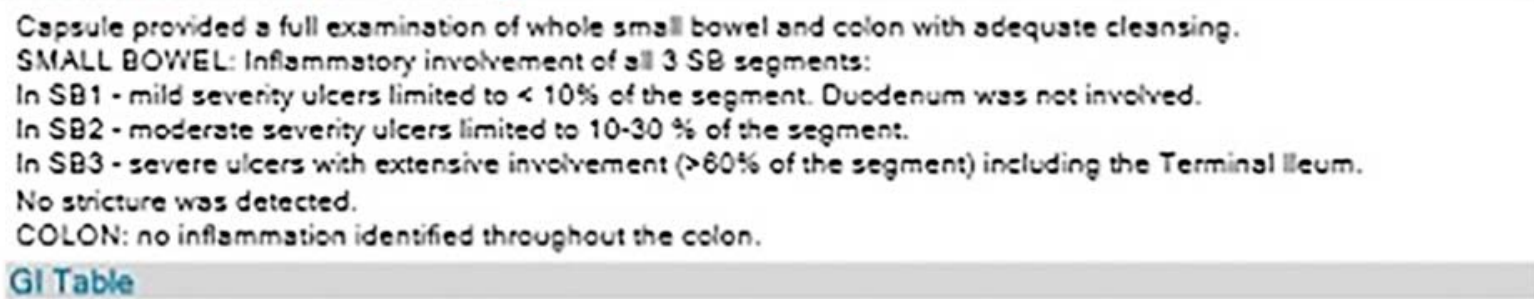

GI Table

$\begin{array}{ll}\text { SEGILENT } & \begin{array}{c}\text { MOST SEVERE } \\ \text { LESION }\end{array} \\ \text { SBI } & 1 \text { - MII } \\ \text { SB II } & 2 \text {-Noderate } \\ \text { SB III } & \text { 3-Severe } \\ \text { Colon } & \text { 0-None }\end{array}$

\section{ST COMONON LESION}

$$
\text { 1. Mild }
$$

2- Moderste

$$
\text { 3-Severe }
$$

0. None

\section{EXTENT \%}

0.10

$10-30$

$00 \cdot 100$

0

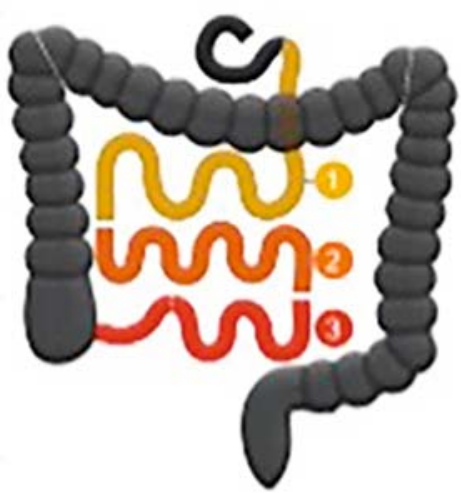

\section{SUB SEGMENTS}

$\begin{array}{llll}\text { Duedenum: } & \text { Not Involved } & \text { Terminal lleum: Involved } \\ \text { Right Colon: } & \text { No: Involved } & \text { Left Colon: } & \text { No: Involved }\end{array}$

\section{Summary and Recommendations}

Complete sssessment of $\mathrm{SB}$ and colen shows diffuse $\mathrm{SB}$ inflummstion, with mos: severe disesse in the termins! ileum. No stricture detested. Consider repesting PlliCsm Crohn's procedure in 6 months.

- Fig. 5 Example of PillCam Crohn's capsule endoscopy report. (Source: Medtronic)

Secondary endpoints included coverage of SB and colon (scale of $1-7$, unconfident to confident), duration of total and segmental reading time (scale of $1-7$, very short to very long), over all video recording quality (scale of $1-7$, poor to excellent), and type, incidence, severity and duration of AEs. Patient satisfaction with the procedure was not assessed.

\section{Statistical and data analysis}

This was a prospective, single-arm, non-blinded feasibility study to assess system functionality of the new PCC platform. No power calculations were made in the determination of sample size. Up to 60 subjects were planned to be enrolled into the study. 
- Table1 Capsule endoscopy bowel preparation outline.

\begin{tabular}{|c|c|c|}
\hline Day & Time & Procedure \\
\hline \multirow[t]{2}{*}{ Day $(-30$ to -1$)$} & All & Clear liquid diet \\
\hline & 19:00-21:00 & 2L PEG/Fortrans/Solucion Bohm \\
\hline \multirow[t]{6}{*}{ Examination Day } & $7: 00-9: 00$ & 2L PEG/Fortrans/Solucion Bohm \\
\hline & 10:00 & PCC ingestion \\
\hline & 11:00 & Optional: $10 \mathrm{mg}$ metoclopramide (only if capsule is in stomach) \\
\hline & Upon SB detection & 0.5 bottle $(88 \mathrm{~mL}$ ) of SUPREP diluted to $240 \mathrm{cc}$ water or 1 sachet of PICO-SALAX $+1 \mathrm{~L}$ water \\
\hline & 3 hr later & $\begin{array}{l}0.5 \text { bottle }(88 \mathrm{~mL}) \text { of SUPREP diluted to } 240 \mathrm{cc} \text { water or } 1 \text { sachet of PICO-SALAX } \\
+1 \mathrm{~L} \text { water }\end{array}$ \\
\hline & 2 hr later & $\begin{array}{l}10 \mathrm{mg} \text { Bisacodyl suppository } / 0.5 \text { bottle }(88 \mathrm{~mL}) \text { of SUPREP diluted to } 240 \mathrm{cc} \text { water or } 1 \text { sachet of } \\
\text { PICO-SALAX according the investigator decision } \\
+1 \mathrm{~L} \text { water }\end{array}$ \\
\hline
\end{tabular}

For the primary objective, system functionality was marked successful if the video was created, the report was generated, and the reader indicated that the report included the information ( 3 points and up of a 5-point scale, coded as "No information" to "Full information") that he/she intended to report on.

For the secondary endpoints, the video coverage (coded as unconfident to confident), reading time (coded as "very short" to "very long") and quality (coded as "poor" to "excellent") were evaluated using a 7-point scale. Summary statistics were provided using standard distribution measures (mean, standard deviation, median, interquartile range [IQR]) along with $95 \%$ confidence interval $(\mathrm{Cl})$ for the mean. For categorical endpoints, the frequency and percent, along with $95 \% \mathrm{Cl}$, were presented.

Subjects who withdrew for any reason except AEs were excluded from the safety analysis. Safety was assessed by characterizing each reported $\mathrm{AE}$ by type, severity, duration, and relationship to the study procedure/device.

Statistical analyses and data management were performed using SAS 9.4 software (SAS Institute Inc., Cary, North Carolina, United States).

\section{Results}

\section{Subject demographics}

Sixty-eight patients with established or suspected IBD were screened. Fourteen subjects (20.6\%) failed screening (unclear gastrointestinal patency). Of the 14 subjects, 13 had established CD and 1 had established ulcerative colitis (UC). A total of 54 subjects were enrolled in the study, of those 41 were included in the final analysis ( $\mathbf{F i g . 4}$ ); five subjects withdrew their consent and eight had procedure-related protocol deviations. The mean age was 40.8 (SD \pm 15.5 ), with $46 \%$ being male and $54 \%$ female ( $\triangleright$ Table 2 ). A summary of patient demographics, baseline data and reason for referral is presented in - Table 2. Of note, of the 29 patients with established CD, 55\% had active disease and of the five patients with UC, $40 \%$ had ac- tive disease. A total of 39 subjects (95\%) had gastrointestinal complaints, and five (12\%) had gastrointestinal (mainly ileo-cecal) resections.

A total of 41 patients were included in the final analysis. A complete summary of SB and/or colon cleansing is described in Table 3. A standard 4-point grading scale system (poor, fair, good, excellent) was used to measure the overall cleansing level. For the SB, cleansing was rated good or excellent in $97.5 \%$ $(40 / 41)$ of subjects, colon cleansing was good or excellent in $75.6 \%(31 / 41)$ of subjects, and the SB and colon cleansing was rated good or excellent in $95.1 \%$ (39/41) of subjects. All capsules reached the toilet with no retention, $83 \%$ while recording (34/41).

For the primary objective ( $\triangleright$ Table 4 ), functionality of the PCC system was rated on a 1 to 5 scale, with no information having a score of 1 and full information having a score of 5 . During video review, one video with problems was encountered (2.44\%; $95 \% \mathrm{Cl}: 0 \%-13.7 \%)$. In this video, segments were not correctly identified, SBI and SBII corresponded the same segment, the capsule did not move, and the system was unable to recognize this. There were no problems during report generation $(0 \% ; 95 \% \mathrm{Cl}: 0 \%-10.2 \%)$ and the report included all intended information (mean score of 5). In addition, all physicians gave the PCC system a high score (5) for information (100\%; $95 \% \mathrm{Cl}: 89.8 \%-100 \%)$.

For secondary objectives ( $\downarrow$ Table 5 ), on a scale of 1-Unconfident to 7-Confident, the PCC system was rated a mean (SD) of 6.7 (0.6) on complete coverage of the SB and a score of 6.1 (1.3) on complete coverage of the colon. A high-level confidence rating (score $5-7$ ) was given for SB and colon coverage (100\%; $95 \% \mathrm{Cl}$ : $89.8 \%-100 \%$ and $90.2 \%$; $95 \% \mathrm{Cl}: 76.9 \%-$ $96.7 \%)$ respectively. Of note, among patients with established $\mathrm{CD}(\mathrm{N}=29), 31 \%$ had proximal involvement $(95 \% \mathrm{Cl}: 17.1 \%-$ $49.4 \%)$.

Video quality of the PCC system was assessed. There were four videos with small interference related to video continuity (gaps) $(9.76 \%$; $95 \% \mathrm{Cl}: 3.3 \%-23.1 \%)$. Overall image quality on 
- Table 2 Summary of demographic data.

\begin{tabular}{|c|c|c|c|c|}
\hline \multicolumn{2}{|l|}{ Parameter } & Statistic & \multicolumn{2}{|c|}{$\begin{array}{l}\text { Overall } \\
N=41\end{array}$} \\
\hline \multicolumn{2}{|l|}{ Age (years) } & $\mathrm{N}$ & \multicolumn{2}{|l|}{41} \\
\hline & & mean $(S D)$ & \multicolumn{2}{|c|}{$40.8(15.5)$} \\
\hline & & median & \multicolumn{2}{|l|}{38} \\
\hline & & $\min ; \max$ & \multicolumn{2}{|c|}{$18 ; 73$} \\
\hline \multicolumn{2}{|l|}{ Gender } & $\mathrm{N}$ & \multicolumn{2}{|l|}{41} \\
\hline - Male & & $\mathrm{n}(\%)$ & 19 & $46.34 \%$ \\
\hline - Female & & $\mathrm{n}(\%)$ & 22 & $53.66 \%$ \\
\hline Referral & & $\mathrm{N}$ & \multicolumn{2}{|l|}{41} \\
\hline - Established CD & & $\mathrm{n}(\%)$ & 29 & $70.73 \%$ \\
\hline - Established UC & & $n(\%)$ & 5 & $12.20 \%$ \\
\hline - Suspected Crohn's & & $\mathrm{n}(\%)$ & 7 & $17.07 \%$ \\
\hline Level of disease & & $\mathrm{N}$ & \multicolumn{2}{|l|}{29} \\
\hline \multirow[t]{2}{*}{ - Established CD } & Active & $\mathrm{n}(\%)$ & 16 & $55.17 \%$ \\
\hline & Quiescent & $\mathrm{n}(\%)$ & 13 & $44.83 \%$ \\
\hline \multicolumn{2}{|l|}{ Level of disease } & $\mathrm{N}$ & \multicolumn{2}{|l|}{5} \\
\hline \multirow[t]{2}{*}{ - Established UC } & Active & $\mathrm{n}(\%)$ & 2 & $40.00 \%$ \\
\hline & Not specified & $n(\%)$ & 3 & $60.00 \%$ \\
\hline
\end{tabular}

- Table 3 Summary of gastrointestinal cleansing.

\begin{tabular}{|l|c|}
\hline Cleansing & Overall $\mathbf{N}=\mathbf{4 1}$ \\
\hline SB cleansing & $0(0 \%)$ \\
\hline Poor & $1(2.44 \%)$ \\
\hline Fair & $16(39.02 \%)$ \\
\hline Good & $24(58.54 \%)$ \\
\hline Excellent & \\
\hline Colon cleansing & $0(0 \%)$ \\
\hline Poor & $10(24.39 \%)$ \\
\hline Fair & $24(58.54 \%)$ \\
\hline Good & $7(17.07 \%)$ \\
\hline Excellent & \\
\hline SB \& colon cleansing & $0(0 \%)$ \\
\hline Poor & $2(4.88 \%)$ \\
\hline Fair & $34(82.93 \%)$ \\
\hline Good & $5(12.2 \%)$ \\
\hline Excellent & \\
\hline SB, small bowel & \\
\hline
\end{tabular}

a scale of 1-Poor to 7-Excellent was given a mean (SD) score of $6.1(0.8)$ with an overall high assessment (score $5-7$ ) of $97.6 \%$ ( $95 \% \mathrm{Cl}: 86.3 \%-100 \%$ ). Reading time experience was rated on a scale of 1 -Very short to 7-Very long. SB reading time was rated a mean (SD) of 3.5 (1.5), colon reading time received a score of 3.6 (1.5), and total video reading time had a score of 3.7 (1.4).

Among the 49 subjects that underwent the PCC procedure, no device-related AEs were reported: 0 (0\%; $95 \% \mathrm{Cl}$ : $0 \%-8.7 \%$ ). There were five procedural-related AEs: one abdominal pain, two cases of vomiting (both withdrew due to noncompliance of the bowel preparation), one sensed weakness and one had nausea. These were considered either mild or moderate AEs, all of which resolved. In one AE case (abdominal pain), an endoscopy was performed which determined that there was no capsule retention; however, the cause could not be elucidated.

\section{Discussion}

$C D$ is a pan-enteric disease that has been long awaiting a technology that will provide useful information about the activity and distribution of the disease in the whole gut in a single study. This five-center prospective feasibility study confirmed that the PCC system is a friendly, minimally invasive system allowing extensive evaluation of the entire bowel. Moreover, when used wisely, it carries minimal risk and no capsule reten- 
- Table4 Summary of primary objectives (subjective assessment questionnaire).

\begin{tabular}{|c|c|c|c|}
\hline \multicolumn{2}{|l|}{ Topic } & \multicolumn{2}{|c|}{ Overall $\mathbf{N}=41$} \\
\hline \multicolumn{4}{|l|}{ System performance assessment } \\
\hline \multicolumn{2}{|l|}{ Problems during the video review } & \multicolumn{2}{|c|}{1 (2.44\%; $95 \% \mathrm{Cl}: 0 \%-13.7 \%)$} \\
\hline \multicolumn{4}{|c|}{$\begin{array}{l}\text { Problems during the video review description: "The segments were not correctly identified. SBI and SBII correspond at the same segment. } \\
\text { The capsule did not move but the system did not recognize that the capsule was not moving" }\end{array}$} \\
\hline \multicolumn{2}{|l|}{ Problems during report generation } & \multicolumn{2}{|c|}{0 (0\%; $95 \% \mathrm{Cl}: 0 \%-10.2 \%)$} \\
\hline \multicolumn{2}{|c|}{ Report included all information that was intended to report on (1-No information 5-Full information) } & \multicolumn{2}{|c|}{$5(0)[5,5-5]$} \\
\hline \multicolumn{2}{|l|}{ High score of information (score 5) } & \multicolumn{2}{|c|}{$41(100 \% ; 95 \% \mathrm{Cl}: 89.8 \%-100 \%)$} \\
\hline Topic & Overall $\mathbf{N}=41$ & & $95 \% \mathrm{Cl}$ for the mean \\
\hline Complete coverage of the SB & $6.7(0.6)[7,5-7]$ & & $6.5-6.9$ \\
\hline High level of confidence on the SB coverage (score 5 - 7) & 41 (100\%; $95 \% \mathrm{Cl}: 89$. & $0 \%)$ & \\
\hline Complete coverage of the colon & $6.1(1.3)[7,2-7]$ & & $5.7-6.5$ \\
\hline High level of confidence on the colon coverage (score $5-7$ ) & 37 (90.2\%; $95 \% \mathrm{Cl}: 76$ & $.7 \%)$ & \\
\hline \multicolumn{4}{|l|}{ Video quality } \\
\hline Malfunctions or interferences related to video/image quality or continuity & \multicolumn{2}{|c|}{4 (9.76\%; $95 \% \mathrm{Cl}: 3.3 \%-23.1 \%)$} & \\
\hline \multicolumn{4}{|l|}{ Details of events: } \\
\hline \multicolumn{4}{|l|}{ - Event 1: Gaps during the last hours of the video } \\
\hline \multicolumn{4}{|l|}{ - Event 2: Three events of temporary break of recorder } \\
\hline \multicolumn{4}{|l|}{ - Event 3: Three events of skip of recording } \\
\hline \multicolumn{4}{|l|}{ - Event 4: Video interruption } \\
\hline Assessment of overall image quality:(1-Poor 7-Excellent) & \multicolumn{2}{|l|}{$6.1(0.8)[6,4-7]$} & $5.9-6.4$ \\
\hline High assessment of overall image quality (score $5-7$ ) & \multicolumn{2}{|c|}{40 (97.6\%; $95 \% \mathrm{Cl}: 86.3 \%-100 \%)$} & \\
\hline \multicolumn{4}{|l|}{ Reading time } \\
\hline SB reading time & $3.5(1.5)[3,1-7]$ & & $3.0-3.9$ \\
\hline Short SB reading experience (score 1 - 3 ) & 21 (51.22\%; $95 \% \mathrm{Cl}: 3$ & $5.8 \%)$ & \\
\hline Colon reading time & $3.6(1.5)[3,1-6]$ & & $3.1-4.1$ \\
\hline Short colon reading experience (score $1-3$ ) & 21 (51.22\%; $95 \% \mathrm{Cl}: 3$ & $5.8 \%)$ & \\
\hline Total Video reading time & $3.7(1.4)[4,1-6]$ & & $3.2-4.1$ \\
\hline Short total video reading experience (score 1 - 3 ) & 19 (46.34\%; $95 \% \mathrm{Cl}: 3$ & $1.3 \%)$ & \\
\hline
\end{tabular}

tions were reported. The capsule and its software were tailored for CD purposes. On one hand, it uses the PCC which is similar to the CC2 system with two cameras, a wider angle of view and an adaptive frame rate, thus allowing for better tissue coverage and longer battery time, but on the other hand, the reading is of the two domes simultaneously with faster frame rate, allowing reading of the entire gut video within 35 minutes (much shorter than that for polyps).

The Rapid 9 software that is used for PCC video evaluation includes relevant, innovative features. Once the first duodenal and first cecal images are identified, the software divides the SB into three tertiles according to their length and not according to transit time as in the previous software. Once the last rectal image is identified, the colon segment is also defined. During video evaluation, at the end of each segment review, the reader is prompted by the system to quickly assess the segment using a more sensible descriptive system: it asks for the grade of the most severe lesion, the most common severity of the lesions and the linear extent of mucosal inflammation in that segment. When taken together over all of the segments, 
this thus results in an assessment of disease severity and burden. All these data are presented in the report as a gastrointestinal table and visually as a gastrointestinal map ( - Fig.5). The software is also designed to create a patient database that is useful during follow-up, providing an effective tool to monitor response to treatment over time. If one takes time and writes the patient's complaints and treatment, all that information will be saved in the computer's hard disc. When consecutive PCC procedures are undertaken for a patient, the extent and severity of SB and colonic involvement can be compared to the previous examinations and according to the different treatments the patient received (i.e. allows assessment of endoscopic response to treatment and monitoring of the patient's whole gut over time) ( $>$ Fig. 2, $>$ Fig. 3).

This first feasibility multicenter trial clearly shows that PCC provides extensive coverage of the pan-enteric gut as intended. As demonstrated by the results, system functionality, entire gut coverage, and video quality were all highly rated by the operators. The reading time was also considered very reasonable and the capsule proved to be very safe with no capsule retention reported among the 49 patients who ingested it.

In the era of mucosal healing and treat to target, the possibility of assessing effect of treatment on both parts of the gut in a relatively friendly manner is of great importance. PCC is a relatively new noninvasive tool that can be used for follow-up of patients who have CD with mucosal inflammation. The PCC system also allows physicians to gather important information that may change our way of treating the patient: the distribution of disease, including proximal bowel involvement (31\% in the current study) which carries a worse prognosis and the state of the mucosa (before and after specific treatment regiments) all in one examination. In their prospective study of 40 patients with CD, D'Haens et al compared the preference of the patients for the colonic capsule versus optical colonoscopy and found that they preferred the capsule procedure [14].

Limitations of our study include, of course that it is a singlearm study with a relatively small sample size $(\mathrm{N}=41)$ with no comparators. Capsule pan-endoscopy is a relatively new concept. Only a few studies have explored the role of a capsule for pan-endoscopy in IBD, adapting the CC2 system for this purpose. This trial provides additional insight into this immerging concept, and raises the cumulative sample size of IBD patients evaluated in these trials to close to 200 [10-13]. All other studies did not have the PillCam v9.0 software but compared the capsule either to ileo-colonoscopy or other modalities, in both adults and children, with good results and no retention or serious AEs $[7,14,16]$. This is one of the few studies to use a specifically designed system to evaluate both the SB and colon. Studies are needed to compare PCC with other imaging modalities, but if we accept that this capsule is at least as good as the SB capsule, then these have already been extensively performed.

\section{Conclusions}

The new PCC system allows for extensive evaluation of the SB and colon. There were no serious AEs that were associated with use of this device in this study, nor was there any capsule retention. This suggests that the PCC endoscopy system is functional and safe in patients with suspected or confirmed IBD.

\section{Acknowledgements}

This study was sponsored and funded by Given Imaging, now a part of Medtronic. Statistical support was provided by Naama Schwartz (Medtronic). Data review was supported by Inbal Eyal (Medtronic). Medical writing and editorial support was provided by Delila Peri (Medtronic).

\section{Competing interests}

Medtronic sponsored the study, provided research support, statistical analysis, and editorial assistance. Drs. Spada, Adler, Fernández-Urién and Eliakim receive consulting fees from Medtronic, Dr. Yanai receives consulting, advisory, lectures and speaker's fees from: Abbvie, Janssen, and Takeda. Dr. Eliakim received a grant from Helmsley Charitable trust, and is on the advisory committee for Photopil I and Tarus Medical. Drs. Lapidus and Eyal are employees of Medtronic.

References

[1] Lichtenstein GR, Hanauer SB, Sandborn W] et al. Management of Crohn's disease in adults. Am J Gastroenterol 2009; 104: 465-483 ; quiz 464,484

[2] Wilkins T, Jarvis K, Patel J. Diagnosis and management of Crohn's disease. Am Fam Physician 2011; 84: 1365 - 1375

[3] Arguelles-Arias F, Rodriguez-Oballe J, Duarte-Chang C et al. Capsule endoscopy in the small bowel Crohn's disease. Gastroenterol Res Pract 2014; 2014: 529136

[4] Park SK, Yang SK, Park SH et al. Long-term prognosis of the jejunal involvement of Crohn's disease. J Clin Gastroenterol 2013; 47: 400 408

[5] Flamant M, Trang C, Maillard O et al. The prevalence and outcome of jejunal lesions visualized by small bowel capsule endoscopy in Crohn's disease. Inflamm Bowel Dis 2013; 19: 1390 - 1396

[6] Cotter J, Dias de Castro F, Moreira M] et al. Tailoring Crohn's disease treatment: the impact of small bowel capsule endoscopy. J Crohns Colitis 2014; 8: 1610-1615

[7] Leighton JA, Helper DJ, Gralnek IM et al. Comparing diagnostic yield of a novel pan-enteric video capsule endoscope with ileocolonoscopy in patients with active Crohn's disease: a feasibility study. Gastrointest Endosc 2017; 85: 196-205.e191

[8] Kopylov U, Yablecovitch D, Lahat A et al. Detection of small bowel mucosal healing and deep remission in patients with known small bowel Crohn's disease using biomarkers, capsule endoscopy, and imaging. Am J Gastroenterol 2015; 110: 1316-1323

[9] Peyrin-Biroulet L, Sandborn W, Sands BE et al. Selecting therapeutic targets in inflammatory bowel disease (STRIDE): determining therapeutic goals for treat-to-target. Am J Gastroenterol 2015; 110 : $1324-1338$

[10] Hausmann J, Schmelz R, Walldorf J et al. Pan-intestinal capsule endoscopy in patients with postoperative Crohn's disease: a pilot study. Scand J Gastroenterol 2017; 52: 1-6 
[11] Hale MF, Drew K, Ejenavi E et al. PWE-029 Colon capsule endoscopy: a useful diagnostic modality that informs patient management. BM] Gut 2015; 64: A223-A224

[12] Hall B, Holleran G, McNamara D. PillCam COLON 2((c)) as a pan-enteroscopic test in Crohn's disease. World J Gastrointest Endosc 2015; 7: $1230-1232$

[13] Boal Carvalho P, Rosa B, Dias de Castro F et al. PillCam COLON 2 in Crohn's disease: A new concept of pan-enteric mucosal healing assessment. World J Gastroenterol 2015; 21: 7233-7241

[14] D'Haens G, Lowenberg M, Samaan MA et al. Safety and feasibility of using the second-generation Pillcam colon capsule to assess active colonic Crohn's disease. Clin Gastroenterol Hepatol 2015; 13: 1480 1486 e1483

[15] Sung J, Ho KY, Chiu HM et al. The use of Pillcam colon capsule in assessing mucosal inflammation in ulcerative colitis: a multicenter study. Endoscopy 2012; 44: $754-758$

[16] Oliva S, Cucchiara S, Civitelli F et al. Colon capsule endoscopy compared with other modalities in the evaluation of pediatric Crohn's disease of the small bowel and colon. Gastrointest Endosc 2016; 83: 975-983

[17] Song HJ, Moon JS, Jeon SR et al. Diagnostic Yield and clinical impact of video capsule endoscopy in patients with chronic diarrhea: a Korean multicenter CAPENTRY study. Gut Liver 2017; 11: 253-260 
- Supplemental Table 1 Study inclusion and exclusion criteria.

\section{Inclusion criteria}

1. Subjects ages $18-75$ years

2. Patient has known CD and/or UC

or

3. Patient suspected to have inflammatory bowel disease and suffers from either:

- Diarrhea for more than 6 weeks and/or

- Abdominal pain for more than 6 weeks and/or

- Extraluminal manifestations of IBD including: erythema nodosum, pyoderma gangrenosum, arthritis, perianal disease, uveitis, aphthous stomatitis

and

4. Suffers from at least one of the symptoms/lab abnormalities listed below:

- Positive inflammatory marker (ESR, CRP, thrombocytosis, fecal lactoferrin, fecal calprotectin) within 3 months prior to enrollment

- Unexplained anemia (less than normal limits) within 3 months prior to enrollment

- Hypoalbuminemia $(<3.5 \mathrm{~g} / \mathrm{dL})$ within 3 months of enrollment

- Positive IBD serology within 3 months of enrollment

- Recurrent fevers

- Unexplained weight loss

- Gastro-intestinal bleeding including melena and/or hematochezia and/or FOBT positive.

- Chronic perianal disease (fistula, fissure, peri-rectal abscess)

- Abnormal imaging of gastrointestinal tract (e. g. MR enterography) suggestive of inflammatory bowel disease

5. For known CD patients, proven patency by the patency capsule or another approach deemed clinically acceptable by the investigator, e. g. CT enterography, MRE performed within the 90 days prior to enrollment.

6. Subject agrees to sign consent form

\section{Exclusion criteria}

1. Antibiotic-associated colitis

2. Stool positive for ova \& parasite and for Clostridium difficile toxin within 3 months of enrollment

3. Other known infectious cause of symptoms

4. Known or suspected intestinal obstruction

5. Nonsteroidal anti-inflammatory drugs (twice weekly or more) during the 4 weeks preceding enrollment.

6. Suspected or known gastrointestinal stricture, followed by patency capsule study or other imaging study that could not prove patency of the gastrointestinal tract

7. Patient is expected to undergo MRI examination within 7 days after ingestion of the capsule

8. Patient with known gastrointestinal motility disorders

9. Subjects with known or suspected delayed gastric emptying

10. Patient suffers from any condition, such as swallowing problems, which precludes compliance with study and/or device instructions

11. Patient has any allergy or other known contraindication or intolerance to the medications used in the study

12. Patient has any condition, which precludes compliance with study and/or device instructions

13. Concurrent participation in another clinical trial using any investigational drug or device

14. Patient suffers from a life-threatening condition

15. Patients with history or clinical evidence of renal disease and/or previous clinically significant laboratory abnormalities of renal function parameters

16. Contraindicated for patients with cardiac pacemakers or other implanted electromedical devices

CD, Crohn's disease; CRP, C-reactive protein; CT, computed tomography; ESR, erythrocyte sedimentation rate; FOBT, fecal occult blood test; IBD, irritable bowel disease; MR, magnetic resonance; MRE, magnetic resonance enterography; MRI; magnetic resonance imaging; UC, ulcerative colitis 